OPEN ACCESS

Edited by:

Mirian A. F. Hayashi,

Federal University of São Paulo, Brazil

Reviewed by:

Lilia Macovei,

The Forsyth Institute, USA

Dinesh Sriramulu,

Shres Consultancy (Life Sciences),

India

*Correspondence.

Ronit Aloni-Grinstein ronitag@iibr.gov.il

Raphael Ber

raphaelb@iibr.gov.il

Specialty section:

This article was submitted to Antimicrobials, Resistance

and Chemotherapy,

a section of the journal

Frontiers in Microbiology

Received: 19 September 2016 Accepted: 15 February 2017

Published: 28 February 2017

Citation:

Aloni-Grinstein R, Schuster O, Yitzhaki S, Aftalion M, Maoz S, Steinberger-Levy I and Ber R (2017)

Isolation of Francisella tularensis

and Yersinia pestis from Blood

Cultures by Plasma Purification and Immunomagnetic Separation Accelerates Antibiotic Susceptibility

Determination

Front. Microbiol. 8:312.

doi: $10.3389 /$ fmicb.2017.00312

\section{Isolation of Francisella tularensis and Yersinia pestis from Blood Cultures by Plasma Purification and Immunomagnetic Separation Accelerates Antibiotic Susceptibility Determination}

\author{
Ronit Aloni-Grinstein ${ }^{1 *}$, Ofir Schuster ${ }^{2}$, Shmuel Yitzhaki ${ }^{2}$, Moshe Aftalion', \\ Sharon Maoz', Ida Steinberger-Levy ${ }^{1}$ and Raphael Ber ${ }^{1 *}$ \\ ${ }^{1}$ Department of Biochemistry and Molecular Genetics, Israel Institute for Biological Research, Ness-Ziona, Israel,
${ }^{2}$ Department of Infectious diseases, Israel Institute for Biological Research, Ness-Ziona, Israel
}

The early symptoms of tularemia and plague, which are caused by Francisella tularensis and Yersinia pestis infection, respectively, are common to other illnesses, resulting in a low index of suspicion among clinicians. Moreover, because these diseases can be treated only with antibiotics, rapid isolation of the bacteria and antibiotic susceptibility testing (AST) are preferable. Blood cultures of patients may serve as a source for bacteria isolation. However, due to the slow growth rates of $F$. tularensis and $Y$. pestis on solid media, isolation by plating blood culture samples on proper agar plates may require several days. Thus, improving the isolation procedure prior to antibiotic susceptibility determination is a major clinically relevant need. In this study, we developed a rapid, selective procedure for the isolation of $F$. tularensis and $Y$. pestis from blood cultures. We examined drop-plating and plasma purification followed by immunomagnetic separation (IMS) as alternative isolation methods. We determined that replacing the classical isolation method with drop-plating is advantageous with respect to time at the expense of specificity. Hence, we also examined isolation by IMS. Sub-localization of $F$. tularensis within blood cultures of infected mice has revealed that the majority of the bacteria are located within the extracellular fraction, in the plasma. Y. pestis also resides within the plasma. Therefore, the plasma fraction was isolated from blood cultures and subjected to an IMS procedure using polyclonal anti-F. tularensis live vaccine strain (LVS) or antiY. pestis antibodies conjugated to $50-\mathrm{nm}$ nano-beads. The time required to reach an inoculum of sufficient bacteria for AST was shortest when using the plasma and IMSs for both bacteria, saving up to 2 days of incubation for F. tularensis and 1 day for Y. pestis. Our isolation procedure provides a proof of concept for the clinical relevance of rapid isolation for AST from F. tularensis- and Y. pestis-infected patients.

Keywords: Francisella tularensis, Yersinia pestis, bacteremia, immunomagnetic separation, antibiotic susceptibility, blood culture 


\section{INTRODUCTION}

Francisella tularensis is a gram-negative facultative intracellular bacterium and the causative agent of tularemia (McCoy and Chapin, 1912). As one of the most infectious pathogenic bacteria known, it is classified by the CDC as a Tier 1 select agent ${ }^{1}$. The disease is endemic in North America and Europe (Sjöstedt, 2007). Disease severity is influenced by the route of infection and the bacterial subtype. The most severe form of tularemia is caused by F. tularensis subsp. tularensis (type A), which is found in North America (Olsufjev and Meshcheryakova, 1983; Staples et al., 2006) whereas F. tularensis subsp. holarctica (type B) is responsible for tularemia across the entire Northern Hemisphere (Jusatz, 1961). F. tularensis live vaccine strain (LVS) (Eigelsbach and Downs, 1961; Tigertt, 1962) is an attenuated type B strain that does not cause disease in humans. LVS is virulent in mice and is thus used in mouse models of the disease (Sjöstedt, 2007). This vaccine has failed to achieve licensing by regulatory authorities (Oyston, 2009). Six clinical forms are observed: ulceroglandular, glandular, oculoglandular, oropharyngeal, typhoid and pneumonic (Anda et al., 2007). The latter is the most relevant in the context of bioterrorism because symptoms develop 3-5 days post-exposure and mortality rates reach $60 \%$ (Gill and Cunha, 1997).

Yersinia pestis is the causative agent of plague, a severe and rapidly progressing disease. Due to its extreme lethality and potential for aerosol transmission, $Y$. pestis is designated by the CDC as a Tier 1 select agent ${ }^{1}$. Symptoms usually begin 1-8 days post-exposure, depending on the route of exposure, infecting strain, disease form (mainly bubonic, septicemic, or pneumonic), dose of infection, and patient immunocompetence (Inglesby et al., 2000). Bacteremia is frequently observed in patients and infected animals (Butler et al., 1976; Perry and Fetherston, 1997). High mortality rates are observed if treatment is not started within 18-24 h after symptom onset (Pollitzer, 1954; Inglesby et al., 2000).

Both tularemia and plague are treated with antibiotics; no safe and efficient vaccines are currently available for tularemia (Boisset et al., 2014) or plague (Pechous et al., 2016). Unfortunately, highly fluoroquinolone-resistant mutants of F. tularensis are easily and quickly obtained (Sutera et al., 2014b), and some exhibit cross-resistance to other clinically relevant antibiotic classes. Similarly, although most naturally occurring $Y$. pestis strains are susceptible to recommended antibiotics, plasmid-mediated single and multiple drug-resistant strains have been isolated from patients (Galimand et al., 2006). Due to the severity of infection by $F$. tularensis or $Y$. pestis and the possibility of acquired resistance to recommended antibiotics (either naturally or intentionally), treatment should not rely solely on organism identification but should include antimicrobial susceptibility testing (AST) of the bacteria isolated from the patient. The conditions for performing AST for F. tularensis or Y. pestis are defined by the CLSI guidelines (CLSI, 2015) and are based on the microdilution technique. Defined concentrations of a bacterial suspension are inoculated into a

${ }^{1}$ https://www.selectagents.gov/selectagentsandtoxinslist.html series of twofold dilutions of the tested antibiotic in a relevant medium and incubated. The Minimal Inhibitory Concentration (MIC) is defined as the lowest antibiotic concentration that completely inhibits bacterial growth, as determined by the unaided eye, following incubation for $24 \mathrm{~h}$ (Y. pestis) or $48 \mathrm{~h}$ (F. tularensis).

Blood cultures are a major source of bacterial isolates for AST. In the past, the isolation of $F$. tularensis from the blood of infected patients has rarely been documented, possibly due to the poor sensitivity of blood culturing systems or the occurrence of bacteremia in the acute stages of infection (Pittman et al., 1977). However, the number of reported tularemia bacteremia cases has increased with the use of improved blood culture isolation systems (Reary and Klotz, 1988), and in recent years, more cases of tularemia bacteremia have been reported in the literature (Haristoy et al., 2003; Sarria et al., 2003; Khoury et al., 2005; Fritzsch and Splettstoesser, 2010; Mohamed et al., 2012; Karagoz et al., 2013; Larssen et al., 2014; Nirkhiwale et al., 2015; Ughetto et al., 2015; Briere et al., 2016). Furthermore, studies have reported bacteremia in mice infected intranasally with $F$. tularensis LVS 48 h post-infection (Chiavolini et al., 2008). The bacteremic phase lasts up to day 7 post-infection. In experimental respiratory tularemia in African green monkeys and cynomolgus macaques, bacteremia was detected 4 days post-infection (Glynn et al., 2015). The clinical significance of $F$. tularensis bacteremia remains unknown; however, bacteremia has been associated with a severe form of the disease and with compromising pneumonia (Karagoz et al., 2013). Bacteremia has also frequently been observed in plague patients (Butler et al., 1976; Perry and Fetherston, 1997).

Although bacteremia has been identified in both diseases, direct transfer of the blood culture into microdilution AST is not feasible, mainly because blood components may interact with either the bacteria or the antibiotics and influence the test results. Moreover, blood components do not allow optical quantification of the bacterial culture. Thus, bacteria isolation by sub-culturing on agar media is a compulsory step that is time-consuming due to the slow growth rates of these bacterial species. For $Y$. pestis, 1-3 days are usually required for the blood culture to be identified as positive, 2 days are required for colony isolation, and another $24 \mathrm{~h}$ are required for AST (Perry and Fetherston, 1997; Inglesby et al., 2000). Due to the slower growth rates of $F$. tularensis, up to 7 days are required for positive blood culture identification (Provenza et al., 1986; Haristoy et al., 2003), 2-3 days of incubation are required for the colony isolation step, and $48 \mathrm{~h}$ are required for AST to determine proper MIC values. In total, 6 days are usually required for $Y$. pestis, and 7 days or more are required until $F$. tularensis blood cultures are identified as positive and bacteria are quantified for AST (Fritzsch and Splettstoesser, 2010; Karagoz et al., 2013; Ughetto et al., 2015). In light of these time intervals, the development of rapid isolation procedures is needed to ensure the clinical relevance of AST results.

Previously, we reported that rapid separation of $Y$. pestis from blood components can be achieved using a serum separation tube (SST) (Steinberger-Levy et al., 2007). In another study, we have 
shown that an immunomagnetic separation (IMS) procedure can be used to pre-enrich $Y$. pestis from environmental samples (Zahavy et al., 2012). To reduce the time interval needed to reach quantified $F$. tularensis and $Y$. pestis cultures suitable for AST, we examined whether the application of an SST and IMS facilitates isolation compared to classic plating or drop-plating directly from blood cultures and whether these procedures can be implemented for the isolation of F. tularensis and Y. pestis from blood cultures.

We first characterized the bacteremic phase in F. tularensisinfected mice and validated ex vivo $F$. tularensis spiking of blood cultures as a mimic of infected blood cultures. Then, we compared the efficacy of the three isolation procedures for both bacterial species. The SST-IMS procedure enabled the isolation of sufficient amounts of $F$. tularensis for AST within the shortest time period for all blood cultures examined, ranging from approximately $10^{3} \mathrm{CFU} / \mathrm{ml}$ to greater than $10^{9} \mathrm{CFU} / \mathrm{ml}$. Moreover, the SST-IMS method was also advantageous for $Y$. pestis, which exhibits faster growth rates than F. tularensis, suggesting that this method may be applicable to a wide range of bacteria.

\section{MATERIALS AND METHODS}

\section{Bacterial Strains, Media, Growth Conditions, and Colony-Forming Unit Determination}

Francisella tularensis LVS (ATCC 29684) was grown on CHA agar (5.1\% cystine heart agar, 1\% hemoglobin), (BD Difco, Sparks, MD, USA) or in cation-adjusted Mueller-Hinton broth (CAMHB) (BBL Difco, Sparks, MD, USA, \#212322) supplemented with $2 \%$ defined growth supplement (IsoVitaleX Enrichment; BBL Difco, Sparks, MD, USA, \#211876) and $3 \mu \mathrm{M}$ hematin (Sigma, Israel \#3281) (termed HLMHI) at $37^{\circ} \mathrm{C}$. The $Y$. pestis vaccine strain EV76 (Girard's strain) (Ben-Gurion and Shafferman, 1981) was grown on BHI-A (brain heart infusion agar, BD Difco, Sparks, MD, USA, \#241830) plates at $28^{\circ} \mathrm{C}$. Colony-forming unit (CFU) counts were determined by plating $100 \mu \mathrm{l}$ of serial 10-fold dilutions in sterile phosphatebuffered saline (PBS, Biological Industries, Beth Haemek, Israel) on CHA and BHI-A plates for F. tularensis and Y. pestis, respectively. Drop-plating was performed by plating $10 \mu \mathrm{l}$ of serial 10-fold dilutions in triplicate on CHA and BHI-A plates.

\section{Animal Infection and Blood Collection}

The animal experiment reported here was approved by the Israel Institute for Biological Research animal care and use committee and was conducted in accordance with the Animal Welfare Act. F. tularensis LVS bacterial cultures were grown to mid-log phase (optical density of $0.1-0.2$ at $660 \mathrm{~nm}$ ) at $37^{\circ} \mathrm{C}$ in TSBC (TSB Difco, Sparks, MD, USA, \#211825 supplemented with $0.1 \%$ cysteine). The bacteria were washed and re-suspended in PBS at the desired concentration. BALB/c female mice (8-10 weeks old, Charles River) were anesthetized with a mixture of $60 \mathrm{mg} / \mathrm{kg}$ ketamine $\mathrm{HCl}$ and $10 \mathrm{mg} / \mathrm{kg}$ xylazine and infected intranasally by application of $25 \mu \mathrm{l}$ containing $10^{5} \mathrm{CFU}$ of $F$. tularensis LVS $\left(10 \mathrm{LD}_{50}\right)$. Mice were monitored daily for clinical symptoms.

At the blood sampling time points, mice were anesthetized with $150 \mathrm{mg} / \mathrm{kg}$ ketamine and $15 \mathrm{mg} / \mathrm{kg}$ xylazine and terminally bled by cardiac puncture using a heparinized syringe. Blood samples were pooled to a final volume of $2-5 \mathrm{ml}$.

\section{Blood Cultures}

Two to five milliliter of fresh infected blood or $10 \mathrm{ml}$ of human blood spiked with bacteria at defined concentrations was incubated in BACTEC Plus Aerobic/F Culture vial (BD, Sparks, MD, USA, \#456005). The blood cultures were shaken at $150 \mathrm{rpm}$ at $37^{\circ} \mathrm{C}$ in a New Brunswick Scientific C76 water bath for the indicated time periods.

\section{Fractionation of Blood Culture Samples}

Blood culture samples were fractionated using SSTs (Vacuette $\mathrm{Z}$ serum Sep. Clot activator, Greiner bio-one, Kremsmunster, Austria \#455071) to obtain the plasma fraction. The sample was loaded into the SSTs and centrifuged for $10 \mathrm{~min}$ at $1700 \mathrm{~g}$ at $20^{\circ} \mathrm{C}$. For $F$. tularensis, the upper fraction was collected, and the bacteria lying on the gel matrix were recovered and added to the plasma fraction. For $Y$. pestis, the upper fraction was discarded, and the bacteria lying on the gel matrix were recovered with $1 \mathrm{ml}$ of PBS. Mononuclear cells were purified from blood culture samples using Vacutainer CPTs (cell preparation tubes) NC (BD, Sparks, MD, USA, \#362781) and Histopaque ${ }^{\circledR}-1077$ gradient (Sigma, Israel \#10771) following the manufacturer's protocols.

\section{Immunomagnetic Separation \\ Preparation of Antibody Nano-Bead Conjugates}

A $50-\mu g$ quantity of polyclonal rabbit anti-F. tularensis LVS serum (Mechaly et al., 2016) or $100 \mu \mathrm{g}$ of polyclonal rabbit anti-Y. pestis Kimberley53 (Zahavy et al., 2012) were covalently linked to $10 \mathrm{mg}$ of $50-\mathrm{nm}$ magnetic beads (fluidMAG-CMX 4106-1, Chemicell, Gmbh, Berlin, Germany) as recommended by the supplier (protocol A11) using EDC (1ethyl-3-[3-dimethylaminopropyl]carbodiimide hydrochloride) (Sigma, Israel \#PG82079) dissolved in MES buffer $(0.1 \mathrm{M}$ 2-(N-morpholino)ethanesulfonic acid), pH 5.

\section{IMS Procedures}

A capture solution containing $80 \mu \mathrm{l}$ of the polyclonal antibodynanoparticle conjugate, $1 \%$ BSA, and $0.5 \%$ Tween 20 was added to the plasma fraction containing the isolated bacteria from the spiked blood cultures. The samples were incubated for $20 \mathrm{~min}$ with gentle rotation at room temperature. LS separation columns (Miltenyi Biotec. Inc, Auburn, CA, USA, \#130-042-401) were placed on the MidiMACS magnetic separator (Miltenyi Biotec Inc, Auburn, CA, USA, \#130-042-302) and preconditioned with $5 \mathrm{ml}$ of PBS. Then, the sample was passed through the column. The column was washed with $3 \mathrm{ml}$ PBS and removed from the magnet, and retained bacteria were washed off with $2 \mathrm{ml}$ of HLMHI for F. tularensis LVS or $1 \mathrm{ml}$ of BHI for Y. pestis EV76 pressed through the column with a piston. The IMS output 
was divided into aliquots of $100 \mu \mathrm{l}$ in a 96-well microtiter plate. Bacteria were grown to O.D. $630 \mathrm{~nm}=0.1$ for F. tularensis, which is equivalent to approximately $1-2 \times 10^{9} \mathrm{CFU} / \mathrm{ml}$, and O.D. $630 \mathrm{~nm}=0.035$ for $Y$. pestis, which is equivalent to $2-3 \times 10^{8}$ $\mathrm{CFU} / \mathrm{ml}$, using O.D.630 $\mathrm{nm}$ at time zero as a blank.

\section{MIC Determination}

Microdilution antibiotic susceptibility standard tests were performed according to the CLSI guidelines for $F$. tularensis and Y. pestis (CLSI, 2015). The recovered bacteria were diluted to yield approximately $1-2 \times 10^{6} \mathrm{CFU} / \mathrm{ml}$ in HLMHI for F. tularensis or approximately $10^{6} \mathrm{CFU} / \mathrm{ml}$ in CAMHB for $Y$. pestis, and $50 \mu \mathrm{l}$ was inoculated in duplicate into 96-well microtiter plates containing $50 \mu \mathrm{l}$ of twofold serial dilutions of each antimicrobial agent in the appropriate medium: doxycycline (Sigma, Israel \#D9891), ciprofloxacin infusion bag (Teva, PetahTikva, Israel). The inoculum size was verified by plating 10 -fold dilutions on $\mathrm{CHA}$ or BHIA and incubation for 3 days at $37^{\circ} \mathrm{C}$ or 2 days at $28^{\circ} \mathrm{C}$ for CFU counts of F. tularensis or Y. pestis, respectively. The microtiter plates were incubated in a plate reader (TECAN Infinite 200) for $48 \mathrm{~h}$ at $37^{\circ} \mathrm{C}$ for $F$. tularensis or $24 \mathrm{~h}$ at $28^{\circ} \mathrm{C}$ for $Y$. pestis in ambient air, and O.D. $630 \mathrm{~nm}$ was read at 1 -h intervals.

Etest assays for $Y$. pestis were conducted on MHA [Mueller Hinton Agar (BBL Difco, Sparks, MD, USA, \#225250)] and on HIMA [MHA supplemented with 0.035\% hemoglobin (BBL Difco, Sparks, MD, USA, \#212392)] and 2\% defined growth supplement (IsoVitaleX Enrichment; BBL Difco, Sparks, MD, USA, \#211876) for F. tularensis. Bacterial cultures with the target turbidity of O.D. $630 \mathrm{~nm}=0.035$ for $Y$. pestis and O.D.630 $\mathrm{nm}=0.1$ for F. tularensis were used as direct inocula by plating $0.1-\mathrm{ml}$ aliquots on the appropriate agar plate and uniformly spreading the bacterial lawn using a Derigalski spatula. Etest strips of doxycycline and ciprofloxacin (bioMerieux, Marcy-L'Etoile, France) were applied on the plated agar and incubated for $24 \mathrm{~h}$ at $28^{\circ} \mathrm{C}$ for $Y$. pestis or $48 \mathrm{~h}$ at $37^{\circ} \mathrm{C}$ for F. tularensis. The MIC was determined by reading the scale values on the strips at the intersection of the growth inhibition zone, according to the manufacturers' instructions.
A

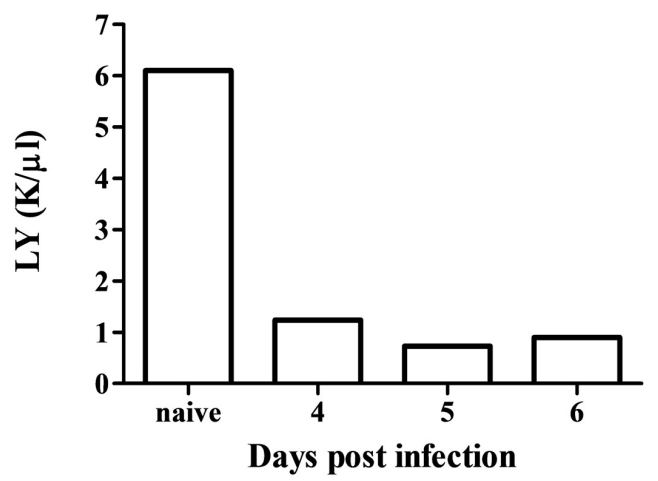

C

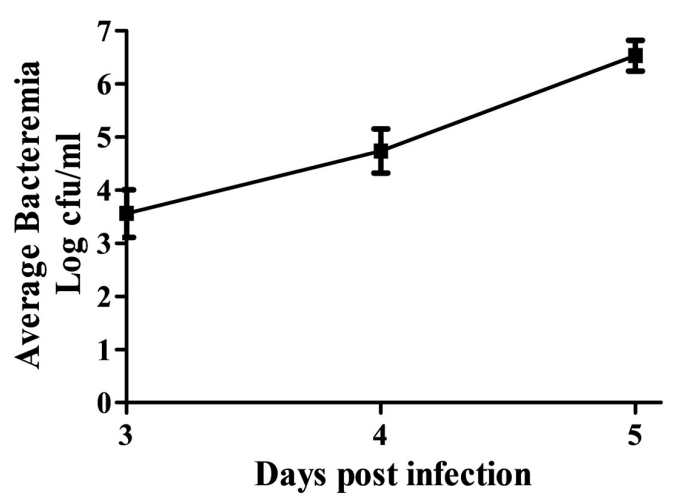

B
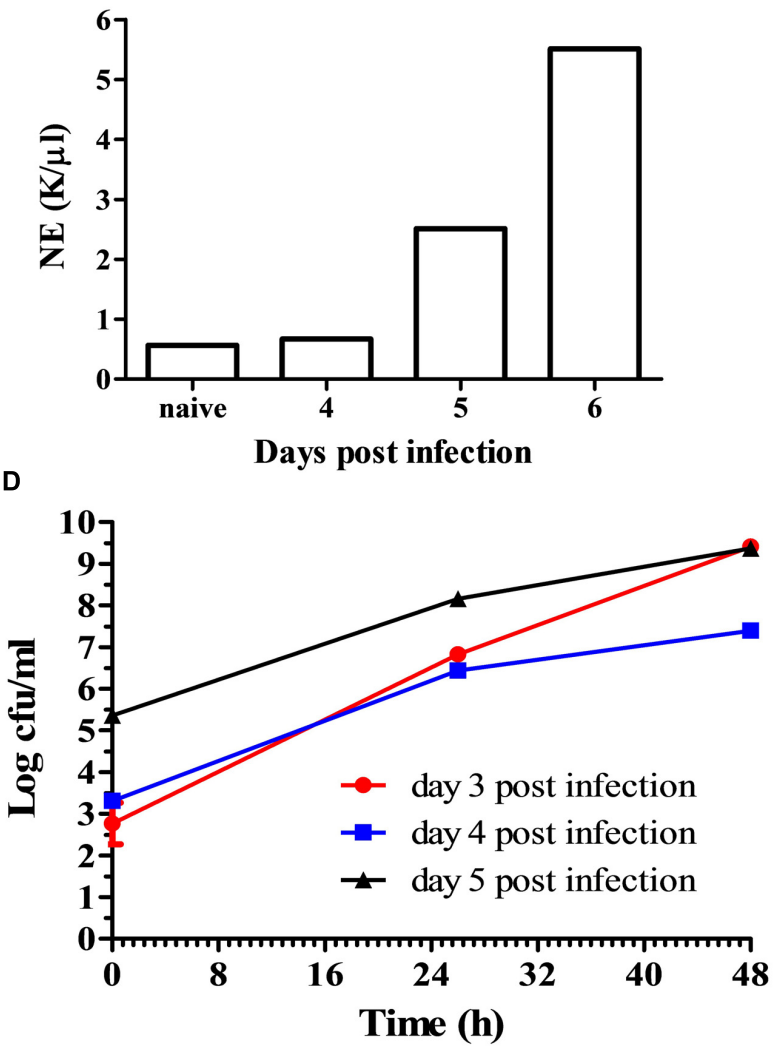

FIGURE 1 | Bacteremia of $\boldsymbol{F}$. tularensis LVS in the blood of infected mice. Mice were infected by the intranasal route with $10^{5}$ CFU per mouse. On days $3-6$ post-infection, groups of 5 mice were terminally bled, and the blood was pooled as one sample. Naïve mice served as controls. Blood samples were subjected to clinical analysis, and disease development was indicated by measuring the levels of lymphocytes (A) and neutrophils (B). Blood samples were introduced into BACTEC Plus Aerobic/F Culture vial. The levels of bacteria in the blood cultures at time $=0 \mathrm{~h}$ were determined by CFU counts and calculated as the in vivo bacteremia levels on days 3, 4, and 5 post-infection (C). F. tularensis LVS multiplication levels within the blood cultures were determined by monitoring growth at $37^{\circ} \mathrm{C}$ under moderate agitation (150 rpm) over $48 \mathrm{~h}$. The red line (circle) represents the blood culture at day 3 post-infection, the blue line (square) represents the blood culture at day 4 post-infection, and the black line (triangle) indicates the blood culture at day 5 post-infection (D). All CFU counts were performed in triplicate. 


\section{RESULTS}

\section{Growth and Sub-localization of F. tularensis in Blood Cultures of Infected Mice}

To develop a rapid isolation process, we first characterized the growth of $F$. tularensis in blood cultures and the sublocalization of the bacteria within the blood culture. BALB/c mice were intranasally infected with $10^{5} \mathrm{CFU}$ of $F$. tularensis LVS. Disease development was monitored by daily clinical and physiological examination. Starting on day 3, blood samples were drawn and pooled from 5 mice per day. Neutrophil and lymphocyte counts of samples from days 4 through 6 were compared to pooled blood samples form control naïve mice. The reduction of lymphocyte levels and elevation of neutrophil levels (Figures 1A,B) indicated an active disease. The pooled blood was spiked into BACTEC Plus Aerobic/F Culture vials. The CFU counts at time $=0 \mathrm{~h}$ in the spiked blood cultures reflected the average in vivo bacteremic state. Bacteremia was evident at day 3 post-infection and its level increased with time, demonstrating the involvement of bacteremia in disease progression within the mice in the intranasal infection model. From the volume of the BACTEC Plus Aerobic/F Culture vial content, we calculated that the average concentration of the bacteria in the blood of the infected mice was approximately $5 \times 10^{3}, 5 \times 10^{4}$, and $5 \times 10^{6} \mathrm{CFU} / \mathrm{ml}$ on days 3, 4, and 5, respectively (Figure 1C). Bacterial growth in the BACTEC Plus Aerobic/F Culture vials were monitored over $48 \mathrm{~h}$. The bacterial load increased by $4-6.5$ orders of magnitude over $48 \mathrm{~h}$, regardless of whether blood was drawn at day 3, 4, or 5 (Figure 1D). These results suggested that intranasal infection of mice with $F$. tularensis LVS recapitulated the bacteremic phase of the disease and that the bacteria in the blood drawn from the infected animals multiplied in the BACTEC Plus Aerobic/F Culture vials.

Francisella tularensis has been reported to reside within blood cells and in the extracellular fraction (Forestal et al., 2007; Yu et al., 2008); thus, it was of interest to examine the distribution of F. tularensis LVS within the blood cultures of the infected animals. We employed the SST previously used to separate Y. pestis from blood cultures (Steinberger-Levy et al., 2007) and separated the plasma fraction from the cellular fraction. At all time points examined, the majority of $F$. tularensis LVS was observed within the plasma fraction (Table 1). Notably, F. tularensis LVS was detected in the plasma fraction immediately after blood was withdrawn from the mice, suggesting that the majority of the bacteria in vivo were also residing in the plasma and not in the intracellular fraction.

\section{Growth and Sub-localization of F. tularensis in Ex vivo Spiked Blood Cultures}

To avoid further infection of animals with $F$. tularensis LVS, we performed ex vivo spiking human blood cultures with the bacterium. To verify that $F$. tularensis in the ex vivo spiked blood cultures also sub-localized in the extracellular fraction, we measured the percentage of plasma-oriented bacteria at various concentrations and time-points post spiking using the SST tubes in a manner identical to that used for bacteria in

TABLE 1 | Localization and growth of $F$. tularensis LVS in blood cultures from infected mice*.

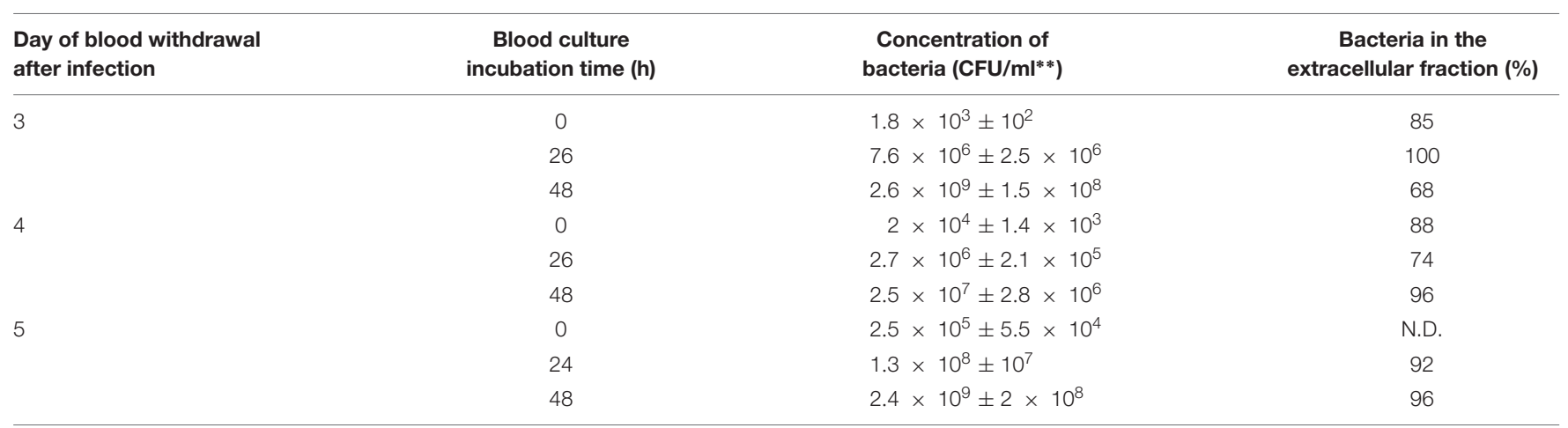

*Each sample contains pooled blood from five infected mice. ** CFU counts were performed in triplicate, and the presented values are the average of the triplicate values. The percent of bacteria in the extracellular fraction was calculated by dividing the CFU counts obtained after separating red blood cells from the plasma fraction using SST by the CFU counts obtained from the blood culture before separation.

TABLE 2 | Levels of $F$, tularensis LVS in the mononuclear and the extracellular fractions of spiked naïve blood cultures grown for 1-2 days.

Concentration of bacteria (CFU/ml*)
Bacteria in mononuclear cells (\%)

Histopaque gradients 10

1.5

16
Bacteria in the extracellular fraction (\%) CPT SST tubes 72

\footnotetext{
*Samples were plated in triplicate for CFU counts.
} 
the blood cultures originating from infected mice (Table 1). Moreover, we quantified the percentage of cells associated with F. tularensis LVS using both Histopaque gradients and CPT tubes to ensure that the majority of the bacteria indeed resided in the extracellular fraction and not in the cellular fraction (Table 2). Similar to the sub-localization in blood cultures from infected mice, the majority of $F$. tularensis LVS was present in the extracellular fraction of the blood in the ex vivo spiked blood cultures. These results confirm that ex vivo spiking of blood cultures is a valid method for developing procedures to improve the isolation of F. tularensis from blood cultures.

\section{Comparison of Methods for Rapid Isolation of $F$. tularensis from Blood Cultures}

\section{Agar Plating and Drop-Plating}

The classic procedure to obtain a bacterial culture from a positive blood culture is the isolation of individual colonies on agar plates. These colonies are then used to prepare a standard inoculum for use in AST. Due to the slow growth rates of F. tularensis LVS strain, 3 days are required to reach colonies of 1-2 mm to yield sufficient bacteria for AST. Thus, we examined whether drop-plating on CHA to contain the plated bacteria in one spot would shorten the incubation time prior to AST of blood cultures when F. tularensis is indicated clinically. For this purpose, blood cultures were spiked with the F. tularensis LVS strain and grown to high densities of approximately $10^{9} \mathrm{CFU} / \mathrm{ml}$. The samples were 10 -fold diluted, and drops of $10 \mu \mathrm{l}$ were plated on CHA plates and incubated for the indicated times at $37^{\circ} \mathrm{C}$ (Figure 2A). Following $24 \mathrm{~h}$ of incubation, F. tularensis LVS drops at $-3 \log$ dilution of the original culture (ending with a concentration of approximately $10^{6} \mathrm{CFU} / \mathrm{ml}$ ) yielded bacterial growth that was sufficient to sample and prepare a standard inoculum for AST assays. Notably, a high drop concentration of $10^{9} \mathrm{CFU} / \mathrm{ml}$ plated directly from the blood culture on $\mathrm{CHA}$ inhibited the growth of $F$. tularensis LVS, probably due to inhibitory factors within the blood culture. Thus, the diluted sample consistently yielded better growth than the non-diluted sample. The 10 -fold dilution of the original sample may have also diluted the inhibitory factors. Sufficient bacterial growth

A
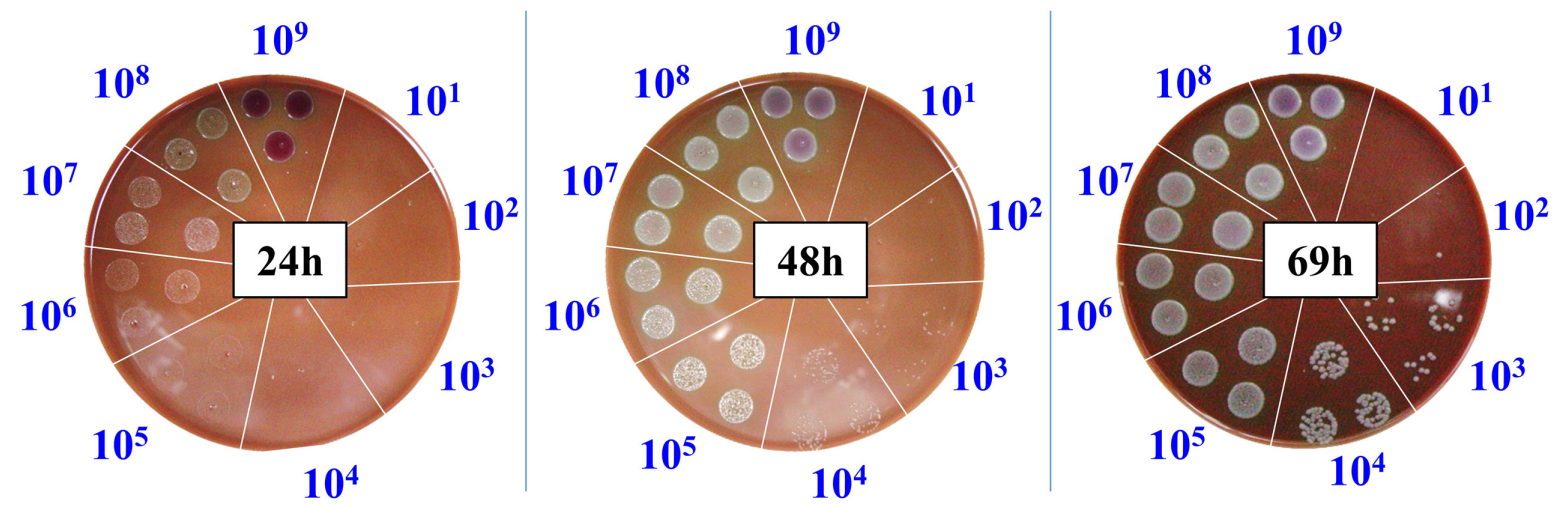

B

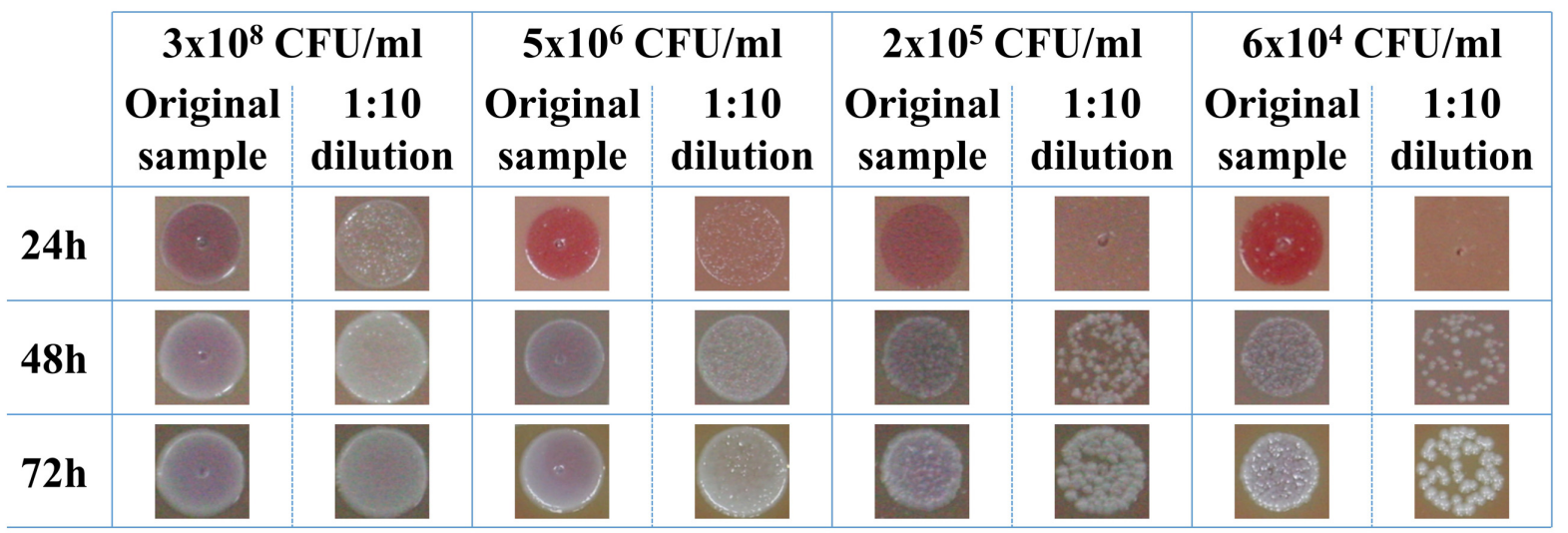

FIGURE 2 | Drop-plating of $\boldsymbol{F}$. tularensis blood culture samples. Blood cultures containing F. tularensis LVS were grown at $37^{\circ} \mathrm{C}$ under moderate agitation (150 rpm) to a concentration of approximately $10^{9} \mathrm{CFU} / \mathrm{ml}$. Both direct and tenfold dilutions were drop-plated as $10 \mu \mathrm{l}$ in triplicate on $\mathrm{CHA}$. The plates were monitored, and growth was recorded at the indicated times (A). Blood cultures of various $F$. tularensis concentrations and their tenfold dilutions were drop-plated. Growth of bacterial lawns and of individual colonies was monitored as a function of time at the indicated incubation time points (B). 
required $48 \mathrm{~h}$ for a blood culture spot sample diluted to a concentration of $10^{4} \mathrm{CFU} / \mathrm{ml}$ and approximately 3 days when the blood culture was diluted to $10^{3} \mathrm{CFU} / \mathrm{ml}$. To ensure that the growth advantage obtained by drop-plating was not merely due to the dilution of the bacteria, which may also dilute growth inhibitors, we applied the drop-plating procedure with spiked blood cultures of different concentrations (Figure 2B). The time schedules obtained for the various blood cultures were within the estimated time frame obtained by the serial dilution of the high-density blood culture (Figure 2A). The estimated required incubation times are summarized in Table 3. In summary, the drop-plating technique may provide an advantage of 1-2 days of incubation compared to classic plating if the blood culture is pure and contains only F. tularensis without other contaminating microorganisms.

\section{Combination of SST and Immunomagnetic Separation of $F$. tularensis LVS from Blood Cultures}

The drop-plating technique reduced the incubation period compared to classic plating, at the expense of a lack of specificity and the potential for a genuine mixed population or fortuitous contamination at blood withdrawal that may remain and be co-cultured in the blood culture. Thus, we used an additional isolation method based on specificity. Of the various available methods, we adopted the SST-IMS procedure. Magnetic beads with a diameter of $50 \mathrm{~nm}$ and coated with primary hyperimmune rabbit anti-F. tularensis serum were used to selectively capture and enrich $F$. tularensis LVS previously purified from blood cultures by SST. The efficacy of the IMS procedure was at least $60 \%$ of the inputs and, in most cases, ranged between 90 and $100 \%$. The IMS outputs were recovered in HLMHI. The growth curves indicated that recovery of the bacteria from the SST-IMS procedure was concentration dependent. We choose O.D.630 $\mathrm{nm}=0.1$ as an endpoint for recovery of F. tularensis LVS because this O.D. reflected mid-log phase at all concentrations examined (data not shown) and a bacterial concentration of approximately $2 \times 10^{9} \mathrm{CFU} / \mathrm{ml}$. Following the establishment and application of the SST-IMS procedure, we determined the correlation between blood culture concentration and the time to obtain sufficient bacteria for downstream AST procedures. To that end, F. tularensis LVS blood cultures

TABLE 3 | Incubation periods required to obtain 1- to 2-mm colonies of F. tularensis LVS following drop-plating as a function of the initial bacterial concentration.

\begin{tabular}{lc}
\hline $\begin{array}{l}\text { Concentration of bacteria in } \\
\text { blood cultures (CFU/ml)* }\end{array}$ & $\begin{array}{c}\text { Estimated time to reach } \\
\text { sufficient growth following } \\
\text { drop-plating (h) }\end{array}$ \\
\hline$\geq 10^{8}$ & $\sim 24$ \\
$10^{7}$ & $\sim 24$ \\
$10^{6}$ & $\sim 24-34$ \\
$10^{5}$ & $\sim 48$ \\
$10^{4}$ & $\sim 48$ \\
$<10^{4}$ & $\sim 72$ \\
\hline
\end{tabular}

*Samples were drop-platted in triplicate as described in Figure 2. ranging from $2.4 \times 10^{3} \mathrm{CFU} / \mathrm{ml}$ to $2.7 \times 10^{9} \mathrm{CFU} / \mathrm{ml}$ were subjected to the SST-IMS procedure. The time required to reach the requested 0.1 O.D.630 nm is presented in Figure 3 and Table 4. Linear regression of $\log$ concentration versus time yielded the prediction equation: Time $(\mathrm{h})=-4.9891 \mathrm{x}$ $\log (\mathrm{CFU} / \mathrm{ml})+46.1$. At all blood culture concentrations examined, the SST-IMS procedure was superior to dropplating with respect to time (Table 3 versus Table 4) and specificity.

\section{Isolation of Y. pestis EV76 from Blood Cultures}

We also examined whether SST-IMS is beneficial for timely isolation of bacteria with a faster growth rate. We chose Y. pestis because it is also designated as a Tier 1 select agent and the time required for its isolation on agar is approximately 2 days. Thus, a quicker isolation step would be clinically valuable. As with $F$. tularensis LVS, we compared the time periods required

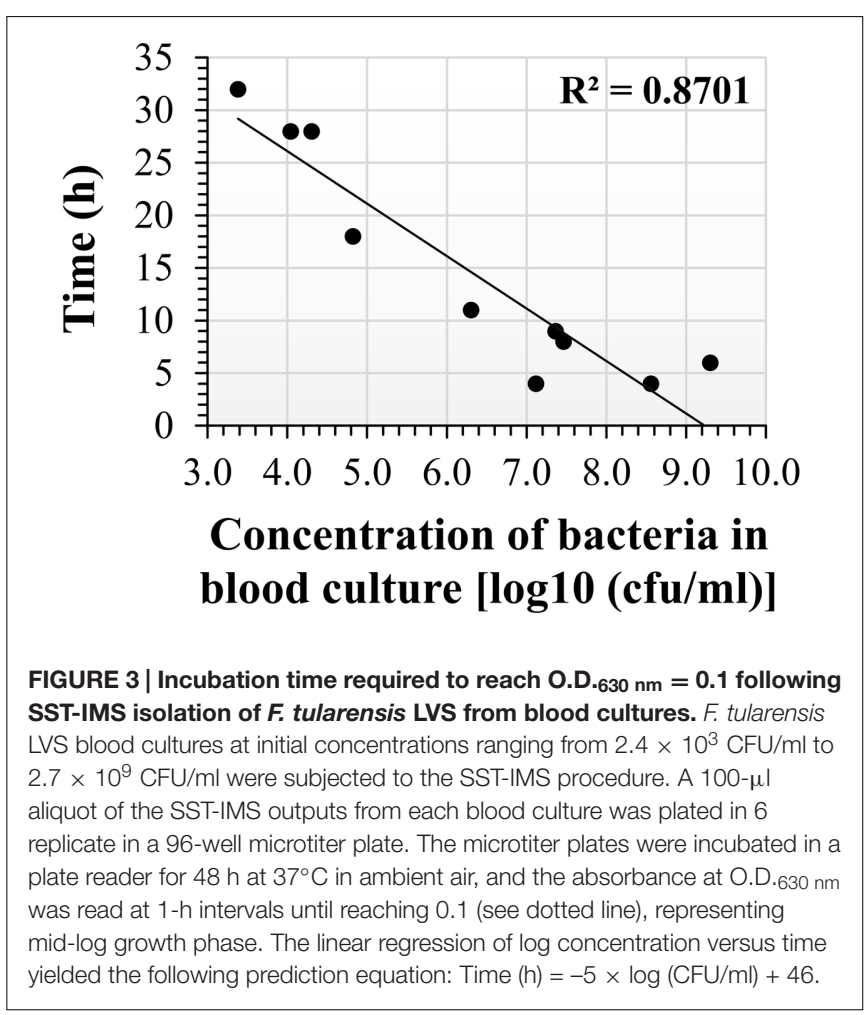

TABLE 4 | Incubation time required to reach O.D. $630 \mathrm{~nm}=0.1$ following SST-IMS isolation of $F$. tularensis LVS from blood cultures.

\begin{tabular}{lc}
$\begin{array}{l}\text { Concentration of bacteria in blood } \\
\text { cultures (CFU/ml) }\end{array}$ & $\begin{array}{c}\text { Estimated time to reach sufficient } \\
\text { growth following SST-IMS (h) }\end{array}$ \\
\hline$\geq 10^{8}$ & $4-6$ \\
$10^{7}$ & $5-10$ \\
$2 \times 10^{6}$ & $\sim 12$ \\
$6.6 \times 10^{4}$ & $\sim 18$ \\
$10^{4}$ & $\sim 28$ \\
$2.4 \times 10^{3}$ & $\sim 33$
\end{tabular}


for classic plating for colony isolation, drop-plating, and SSTIMS for the isolation of $Y$. pestis EV76 from spiked blood cultures. Two incubation days are required for classic isolation to obtain isolated colonies at any given bacterial concentration. No bacteria were observed on BHIA plates $24 \mathrm{~h}$ after drop-plating a blood culture of $2 \times 10^{3} \mathrm{CFU} / \mathrm{ml}$ (Figure 4). Colony formation required $48 \mathrm{~h}$ following drop-plating (data not shown). However, at concentrations of $2.5 \times 10^{5} \mathrm{CFU} / \mathrm{ml}$ and greater, colonies were observed after only $24 \mathrm{~h}$ of incubation (Figure 4). Thus, the dropplating technique may provide an advantage of a day compared to classic plating, although at the expense of selectivity. Based on our previous work (Steinberger-Levy et al., 2007), Y. pestis EV76 was isolated from the plasma fraction of the blood culture sample using SST and subjected to an IMS procedure using 50$\mathrm{nm}$ magnetic beads coated with primary hyperimmune rabbit anti-Y. pestis serum. The efficacy of the IMS procedure was at least $60 \%$ of the inputs and ranged between 90 and $100 \%$ in most cases. Bacteria were recovered in BHI medium until reaching O.D.630 $\mathrm{nm}=0.035$, reflecting mid-log growth (data not shown) and a bacterial concentration of $2-3 \times 10^{8} \mathrm{CFU} / \mathrm{ml}$. The time required to reach O.D.630 nm $=0.035$ as a function of bacterial concentration is presented in Figure 5. Linear regression of log concentration versus time yielded the following prediction equation: Time $(\mathrm{h})=-6.1926 \times \log (\mathrm{CFU} / \mathrm{ml})+52.3$. Consistent with the results obtained for F. tularensis LVS, SSTIMS was superior to the classic and drop-plating procedures for Y. pestis EV76 (Table 5). Our results suggest that the SST-IMS procedure is an efficient and prompt procedure that may be used for rapid isolation of the Tier 1 agents $F$. tularensis and Y. pestis.

\section{MIC Determination}

The recommended antibiotics for post-exposure prophylaxis of $F$. tularensis and $Y$. pestis are ciprofloxacin and doxycycline (Inglesby et al., 2000; Dennis et al., 2001). As a proof of concept of the validity of our isolation procedure, we performed

\begin{tabular}{|c|c|c|}
\hline & $\begin{array}{l}\text { Original } \\
\text { sample }\end{array}$ & $\begin{array}{c}1: 10 \\
\text { dilution }\end{array}$ \\
\hline \multicolumn{3}{|l|}{$2 \times 10^{3} \mathrm{cfu} / \mathrm{ml}$} \\
\hline \multicolumn{3}{|l|}{$2.5 \times 10^{5} \mathrm{cfu} / \mathrm{ml}$} \\
\hline \multicolumn{3}{|l|}{$2.6 \times 10^{6} \mathrm{cfu} / \mathrm{ml}$} \\
\hline $\begin{array}{l}\text { GURE } 4 \text { | Drop-plating of } \mathbf{Y} . \mathbf{p e} \\
\mathbf{1 7 6} \text { blood cultures were grown a } \\
2.6 \times 10^{6}, 2.5 \times 10^{5} \text {, and } 2 \times \\
\text { lutions were drop-plated in triplic } \\
\text { ere incubated at } 28^{\circ} \mathrm{C} \text {, and grow }\end{array}$ & $\begin{array}{l}\text { stis blood culture } \\
37^{\circ} \mathrm{C} \text { under moder } \\
0^{3} \mathrm{CFU} / \mathrm{ml} \text {. Both dir } \\
\text { te as } 10-\mu / \text { aliquots }\end{array}$ & $\begin{array}{l}\text { samples. Y. pestis } \\
\text { ate agitation (150 rpm } \\
\text { rect and } 10 \text {-fold } \\
\text { on BHIA. The plates } \\
\text { h post plating. }\end{array}$ \\
\hline
\end{tabular}

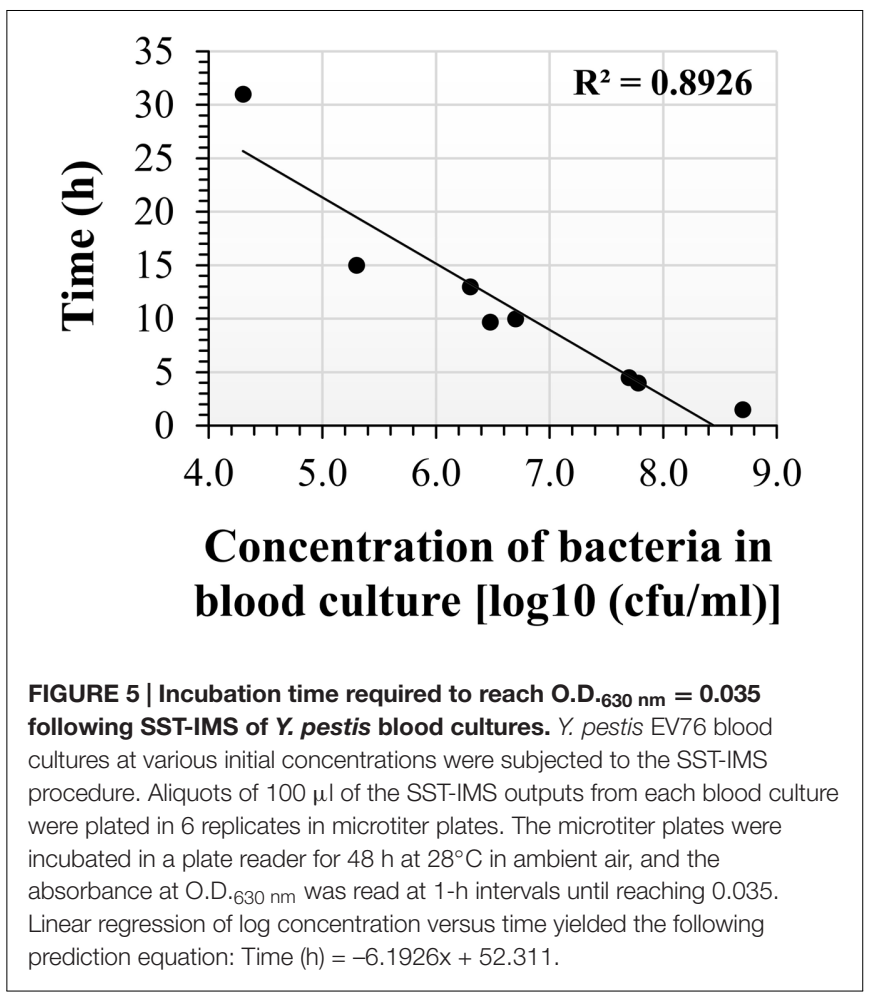

TABLE 5 | Comparison of the time schedules for the isolation of $Y$. pestis EV76 from blood cultures by classic plating, drop-plating and SST-IMS.

Concentration of bacteria in blood cultures (CFU/ml)

Incubation time (h)

\begin{tabular}{lccc}
\cline { 2 - 4 } & Classic plating & Drop-plating* & SST-IMS \\
\hline $10^{7}$ & $\sim 48$ & $\sim 24$ & $\sim 8$ \\
$2.6 \times 10^{6}$ & $\sim 48$ & $\sim 24$ & $\sim 13$ \\
$2.5 \times 10^{5}$ & $\sim 48$ & $\sim 24$ & $\sim 20$ \\
$2 \times 10^{4}$ & $\sim 48$ & $\sim 24$ & $\sim 31$ \\
\hline
\end{tabular}

*Samples were drop-plated in triplicate.

AST of these recommended antibiotics. F. tularensis cultures in HLMHI that reached O.D. $630 \mathrm{~nm}=0.1$ (reflecting approximately $2 \times 10^{9} \mathrm{CFU} / \mathrm{ml}$ ) and $Y$. pestis cultures in BHI that reached O.D. $630 \mathrm{~nm}=0.035$ (reflecting approximately $2-3 \times 10^{8} \mathrm{CFU} / \mathrm{ml}$ ) were diluted as required by the CLSI to yield approximately $5 \times 10^{5}-2 \times 10^{6} \mathrm{CFU} / \mathrm{ml}$ and inoculated into 96-well microtiter plates in a final volume of $100 \mu \mathrm{l} /$ well. Microdilution tests were performed as described in the Section "Materials and Methods." The MIC was defined at the end of the incubation time ( $24 \mathrm{~h}$ for $Y$. pestis and $48 \mathrm{~h}$ for F. tularensis) as the lowest concentration that reduced growth to less than $10 \%$ of the O.D.630 nm of the growth control, and a lack of visible growth was verified by the unaided eye. MIC values are presented as a range and include all values obtained in repetitions $(n=3)$ (Table 6). F. tularensis LVS displayed the expected 'susceptible' category as indicated by MICs of $0.016 \mu \mathrm{g} / \mathrm{ml}$ for ciprofloxacin, $\leq 0.5 \mu \mathrm{g} / \mathrm{ml}$ (the endpoint for the susceptible category for this bacterium), and $0.06-0.125 \mu \mathrm{g} / \mathrm{ml}$ for doxycycline, $\leq 4 \mu \mathrm{g} / \mathrm{ml}$. Similar MIC ranges 
were obtained by the Etest method, which does not appear to have an advantage over microdilution with respect to the required incubation period but may prove useful for low levels of contamination in blood cultures. Similarly, microdilution tests $(n=3)$ of $Y$. pestis EV76 revealed MIC values of $0.5-1 \mu \mathrm{g} / \mathrm{ml}$ for doxycycline and $0.016-0.032 \mu \mathrm{g} / \mathrm{ml}$ for ciprofloxacin. In the Etests, the MIC values were similar and ranged from 0.5 to $0.75 \mu \mathrm{g} / \mathrm{ml}$ for doxycycline and 0.023 to $0.032 \mu \mathrm{g} / \mathrm{ml}$ for ciprofloxacin. All MIC results were in complete agreement with assays conducted using standard inoculum and test conditions. These MIC results indicate that the SST-IMS procedure is suitable for the rapid isolation of $F$. tularensis and Y. pestis for AST.

\section{DISCUSSION}

The symptoms of tularemia include fever, headache, chills, sore throat, and body aches (Dennis et al., 2001). These symptoms are common to other illnesses, resulting in a low index of suspicion for tularemia among clinicians. Tularemia may be
TABLE 6 | Minimal Inhibitory Concentration (MIC) determination.

\begin{tabular}{lcccccc}
\hline & \multicolumn{2}{c}{ Doxycycline $(\mu \mathrm{g} / \mathrm{ml})$} & & \multicolumn{2}{c}{ Ciprofloxacin $(\mu \mathrm{g} / \mathrm{ml})$} \\
\cline { 2 - 3 } & Microdilution & Etest & & Microdilution & Etest \\
\hline F. tularensis LVS & $\begin{array}{c}0.06-0.125 \\
\text { Y. pestis EV76 }\end{array}$ & $0.5-1$ & $0.5-0.75$ & & $0.016-0.032$ & $0.023-0.032$ \\
\hline
\end{tabular}

considered an option only after exclusion of more common infectious diseases. Consequently, some tularemia patients may be treated with broad-range antibiotics without actual identification of F. tularensis as the causative agent (Fritzsch and Splettstoesser, 2010). Thus, isolation of the bacteria may benefit both identification and, more importantly, AST characterization to assure proper antibiotic treatment. In recent years, efforts have been made to facilitate AST procedures for tularemia and develop new approaches to determine susceptibility toward both the extracellular and intracellular forms of the bacteria (Sutera et al., 2014a; Aloni-Grinstein et al., 2015).

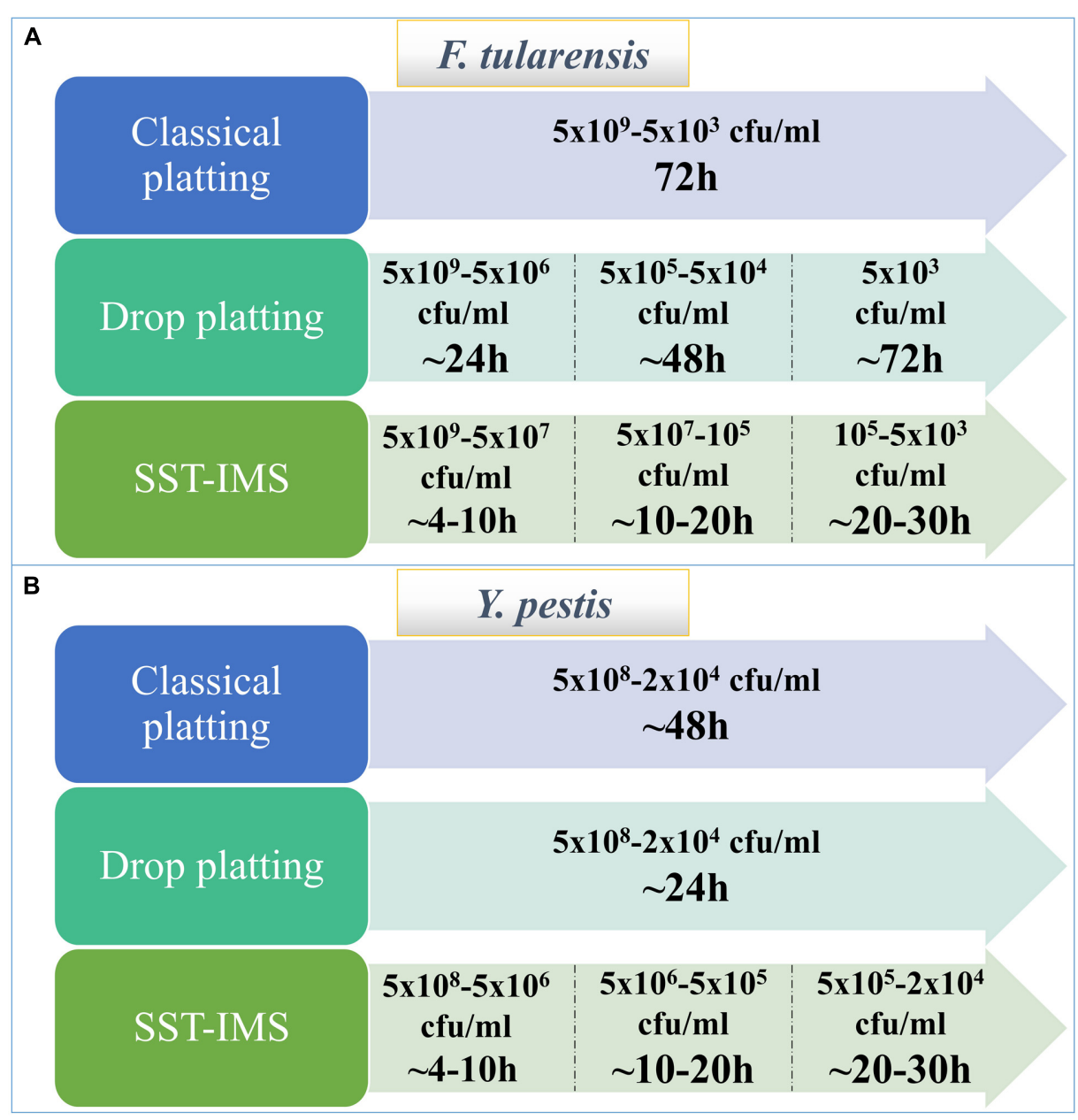

FIGURE 6 | Time required to reach the required inoculum of bacteria for AST using the different isolation methods. F. tularensis LVS (A). Y. pestis EV76 (B). 
Blood cultures are a potential clinical source of bacteria from the patient. Moreover, determination of bacteremia may have an impact on the clinical outcome and medical consideration toward the patient. For years, bacteremia was rarely identified, but new culture systems have improved growth and detection, and bacteremia is now reported more widely. Indeed, we observed the development of bacteremia in our mouse model during the progression of the disease (Figure 1) and determined that the majority of the bacteria were in the plasma fraction rather than the cellular fraction (Table 1). In an attempt to mimic blood cultures of infected animals, we ex vivo spiked $F$. tularensis LVS into BACTEC Plus Aerobic/F Culture vials containing naïve blood and showed that the bacteria reside mainly in the plasma fraction in spiked blood cultures as well (Table 2). This behavior allowed us to develop a rapid isolation procedure for bacteria from blood cultures.

The isolation of $F$. tularensis from blood cultures is a tedious procedure. Blood culture components negatively influence both PCR identification procedures (Regan et al., 2012) and the prerequisite quantification step and inoculum size adjustment using absorbance determination for standard AST (CLSI, 2015). Thus, sub-culturing is required prior to AST. Due to the slow growth rates of $F$. tularensis and $Y$. pestis, sub-culturing for isolation is time-consuming. Here, we aimed to bypass the subculturing isolation step by either drop-plating or by physical separation from red blood cells and an IMS procedure for transferring the bacteria from the plasma to rich liquid medium. Indeed, drop-plating blood cultures with $F$. tularensis LVS with at least $10^{6} \mathrm{CFU} / \mathrm{ml}$ (Figure 2) and Y. pestis with at least $10^{5}$ $\mathrm{CFU} / \mathrm{ml}$ (Figure 4) yielded sufficient bacteria by $24 \mathrm{~h}$. However, small percentages of these samples may be contaminated because no selective procedure was applied to distinguish F. tularensis or $Y$. pestis from potential contaminating bacteria due to either natural infection or contamination during the blood withdrawal procedure (Weinstein, 2003). This drawback highlights the advantage of IMS, which enriches bacteria by selective immunoseparation. The presence of $F$. tularensis LVS bacteria primarily in the extracellular fraction in blood cultures from infected animals allowed us to obtain the majority of the bacteria from the blood culture by a simple step using SSTs before IMS purification. IMS separates the

\section{REFERENCES}

Aloni-Grinstein, R., Shifman, O., Lazar, S., Stienberger-Levy, I., Maoz, S., and Ber, R. (2015). A rapid real-time quantitiative PCR assay to determine the minimal inhibitory extracellular concentration of antibiotics against and intracellular Francisella tularensis Live Vaccine Strain. Front. Microbiol. 6:1213. doi: $10.3389 /$ fmicb. 2015.01213

Anda, P., Pearson, A. D., and Tarnvik, A. (2007). WHO Guidelines on Tularaemia. Geneva: World Health Organization.

Ben-Gurion, R., and Shafferman, A. (1981). Essential virulence determinants of different Yersinia species are carried on a common plasmid. Plasmid 5, 183-187. doi: 10.1016/0147-619X(81)90019-6

Boisset, S., Caspar, Y., Sutera, V., and Maurin, M. (2014). New therapeutic approaches for treatment of tularemia: a review. Front. Cell. Infect. Microbiol. 4:40. doi: 10.3389/fcimb.2014.00040 bacteria from the blood components and growth-inhibitory substances present in the blood culture and components that mask proper quantification by turbidity measurement. The recovered concentrated bacteria in enrichment media permit rapid growth to the essential load necessary for use as an inoculum for various AST methods. Indeed, we have shown that the isolation of bacteria by the SST-IMS procedure permits proper MIC determination by microdilution within a shorter time than the conventional isolation method. Moreover, we have shown that this procedure is superior to classic plating for both $F$. tularensis and $Y$. pestis (Figure 6), which represent bacteria with different growth rates. These different bacteria validate the universality of the method for other bacteremia-causing bacteria. In summary, we have shown that the SST-IMS procedure is a rapid, specific procedure for the isolation of bacteria from positive blood cultures. The SST-IMS procedure allows AST for both $F$. tularensis and $Y$. pestis to be performed within a significantly shorter time period after the blood culture is identified as positive for these pathogens (Tables 3-5), thus shortening the time necessary to conduct AST for proper antibiotic treatment determination.

\section{AUTHOR CONTRIBUTIONS}

Research project design: RA-G, OS, SY, IS-L, and RB. Experiments: RA-G, OS, SY, MA, SM, and RB. Writing: RA-G and RB.

\section{FUNDING}

This study was supported by the Israel Institute of Biology Research, Ness-Ziona, Israel.

\section{ACKNOWLEDGMENTS}

We thank Erez Bar-Haim and Hila Cohen for infecting the animals and Yossi Shlomovitz for assisting with animal work and blood withdrawal.

Briere, M., Kaladji, A., Douane, F., Breux, J. P., Touroult-Jupin, P., Boisset, S., et al. (2016). Francisella tularensis aortitis. Infection 44, 263-265. doi: 10.1007/ s15010-015-0824-4

Butler, T., Levine, J., Linh, N., Chau, D., Adickman, M., and Arnold, K. (1976). Yersinia pestis infection in Vietnam II: quantitative blood cultures and detection of endotoxin in the cerebrospinal fluid of patients with meningitis. J. Infect. Dis. 133, 493-498. doi: 10.1093/infdis/133. 5.493

Chiavolini, D., Alroy, J., King, C. A., Jorth, P., Weir, S., Madico, G., et al. (2008). Identification of immunologic and pathologic parameters of death versus survival in respiratory tularemia. Infect. Immun. 76, 486-496. doi: 10.1128/IAI. 00862-07

CLSI (2015). Methods for Antimicrobial Dilution and Disk Susceptibility Testing of Infrequently Isolated or Fastidious Bacteria, 3rd Edn. Wayne, PA: Clinical and Laboratory Standards Institute. 
Dennis, D. T., Inglesby, T. V., Henderson, D. A., Bartlett, J. G., Ascher, M. S., Eitzen, E., et al. (2001). Tularemia as a biological weapon: medical and public health management. JAMA 285, 2763-2773. doi: 10.1001/jama.285.21.2763

Eigelsbach, H. T., and Downs, C. M. (1961). Prophylactic effectiveness of live and killed vaccines. 1 . Production of vaccine and evaluation in the white mouse and guinea pig. J. Immunol. 87, 415-425.

Forestal, C. A., Malik, M., Scatlett, S. V., Savitt, A. G., Bennach, J. L., Sellati, T. J., et al. (2007). Francisella tularensis has a significant extracellular phase in infected mice. J. Infect. Dis. 196, 134-137. doi: 10.1086/518611

Fritzsch, J., and Splettstoesser, W. D. (2010). Septic pneumonic tularemia caused by Francisella tularensis subsp. holarctica biovar II. J. Med. Microbiol. 59, 1123-1125. doi: 10.1099/jmm.0.019893-0

Galimand, M., Carniel, E., and Courvalin, P. (2006). Resistance of Yersinia pestis to antimicrobial agents. Antimicrob. Agents Chemother. 50, 3233-3236. doi: 10.1128/AAC.00306-06

Gill, V., and Cunha, B. A. (1997). Tularemia pneumonia. Semin. Respir. Infect. 12, 61-67.

Glynn, A. R., Alves, D. A., Frick, O., Erwin-Cohen, R., Porter, A., Norris, S., et al. (2015). Comparison of experimental respiratory tularemia in threee nonhuman primate species. Comp. Immunol. Microbiol. Infect. Dis. 39, 13-24. doi: 10.1016/ j.cimid.2015.01.003

Haristoy, X., Lonzniewski, A., Tram, C., Simeon, D., Bevanger, L., and Lion, C. (2003). Francisella tularensis bacteremia. J. Clin. Microbiol. 41, 2774-2776. doi: 10.1128/JCM.41.6.2774-2776.2003

Inglesby, T. V., Dennis, D. T., Henderson, D. A., Bartlett, J. G., Ascher, M. S., Eitzen, E., et al. (2000). Plague as a biological weapon. Medical and public health management. JAMA 283, 2281-2290. doi: 10.1001/jama.283.17.2281

Jusatz, H. J. (1961). The Geographical Disribution of Tularemia Throughout the World 1911-1959. Hamburg: Federal Republic of German.

Karagoz, S., Kilic, S., Berk, E., Uzel, A., Celebri, B., Comoglu, S., et al. (2013). Francisella tularensis bacteremia: report of two cases and review of the literature. New Microbiol. 36, 315-323.

Khoury, J. A., Bohl, D. L., Hersh, M. J., Argoudelis, A. C., and Brennan, D. C. (2005). Tularemia in a kidney transplant recipient: an unsuspected case and literature review. Am. J. Kidney Dis. 45, 926-929. doi: 10.1053/j.ajkd.2005. 02.006

Larssen, K. W., Bergh, K., Heier, B. T., Vold, L., and Afset, J. E. (2014). All-time high tularemia incidence in Norway in 2011: report from the national surveillance. Eur. J. Clin. Microbiol. Infect. Dis. 33, 1919-1926. doi: 10.1007/s10096-0142163-2

McCoy, G. W., and Chapin, C. W. (1912). Further observation on a plaguelike disease of rodents with a preliminary note on causative agent, Bacterium tularense. J. Infect. Dis. 10, 61-72. doi: 10.1093/infdis/10.1.61

Mechaly, A., Cohen, H., Cohen, O., and Mazor, O. (2016). A biolayer interfermetrybased assay for the rapid and highly senitive detection of biowarfare agents. Anal. Biochem. 506, 22-27. doi: 10.1016/j.ab.2016.04.018

Mohamed, S. E. R., Mubarak, A. I., and Alfarooq, L. O. (2012). Francisella tularensis bacteremia: a case report from Sudan. Case Rep. Infect. Dis. 2012:405737. doi: $10.1155 / 2012 / 405737$

Nirkhiwale, S. D., Gehlot, G. S., Bandi, A. K., and Jasani, A. N. (2015). Isolation of Francisella tularensis from blood culture. Indian J. Med. Microbiol. 33, 329-331. doi: 10.4103/0255-0857.154901

Olsufjev, N. G., and Meshcheryakova, I. S. (1983). Subspecific taxonomy of Francisella tularensis McCoy and Chapin, 1912. Int. J. Syst. Bacteriol. 33, 872-874. doi: 10.1099/00207713-33-4-872

Oyston, P. C. (2009). Francisella tularensis vaccines. Vaccine 27, D48-D51. doi: 10.1016/j.vaccine.2009.07.090

Pechous, R. D., Sivaraman, V., Stasuli, N. M., and Goldman, W. E. (2016). Pneumonic Plague: the darker side of yersinia pestis. Trends Microbiol. 24, 190-197. doi: 10.1016/j.tim.2015.11.008

Perry, R. D., and Fetherston, J. D. (1997). Yersinia pestis-etiologic agent of plague. Clin. Microbiol. Rev. 10, 35-66.
Pittman, B., Shaw, E. B. J., and Cherry, W. B. (1977). Isolation of Francisella tularensis from infected frozen human blood. J. Clin. Microbiol. 5, 621-624.

Pollitzer, R. (1954). Plague. WHO Monograph Series 22. Geneva: WHO.

Provenza, J. M., Klotz, S. A., and Penn, R. L. (1986). Isolation of Francisella tularensis from blood. J. Clin. Microbiol. 24, 453-455.

Reary, B. W., and Klotz, S. A. (1988). Ehancing recovery of Francisella tularensis from blood. Diagn. Microbol. Infect. Dis. 11, 117-119. doi: 10.1016/07328893(88)90080-6

Regan, J. F., Furtado, M. R., Brevnov, M. G., and Jordan, J. A. (2012). A sample extraction method for faster, more sensative PCR-Based detection of pathogens in blood culture. J. Mol. Diagn. 14, 120-129. doi: 10.1016/j.jmoldx.2011.10.001

Sarria, J. C., Vidal, A. M., Kimbrough, R. C., and Figueroa, J. E. (2003). Fatal infection caused by Francisells tularensis in a neutropenic bone marrow transplant recipient. Ann. Hematol. 82, 41-43.

Sjöstedt, A. (2007). Tularemia: history, epidemiology, pathogen physiology and clinical manifestations. Ann. N. Y. Acad. Sci. 1105, 1-29. doi: 10.1196/annals. 1409.009

Staples, J. E. K., Kubota, K. A., Chalcraft, L. G., Mead, P. S., and Peterson, J. M. (2006). Epidemiologic and molecular analysis of human tularemia, United States 1964-2004. Emerg. Infect. Dis. 12, 1113-1118. doi: 10.3201/eid1207. 051504

Steinberger-Levy, I., Zahavy, E., Cohen, S., Flashner, Y., Mamroud, E., Aftalion, M., et al. (2007). Enrichment of Yersinia pestis from blood cultures enables rapid antimicrobial susceptibility determination by flow cytometry. Adv. Exp. Med. Biol. 603, 339-350. doi: 10.1007/978-0-387-72124-8\_31

Sutera, V., Caspar, Y., Boisset, S., and Maurin, M. (2014a). A new dye uptake assay to test the activity of antibiotics against intracellular Francisella tularensis. Front. Cell. Infect. Microbiol. 4:36. doi: 10.3389/fcimb.2014. 00036

Sutera, V., Levert, M., Burmeister, W. P., Schneider, D., and Maurin, M. (2014b). Evolution towards high-level fluoroquinolone resistance in Francisella species. J. Antimicrob. Chemother. 69, 101-110. doi: 10.1093/jac/dkt321

Tigertt, W. D. (1962). Soviet viable Pasteurella tularensis vaccines: a review of selected articles. Bacteriol. Rev. 26, 354-373.

Ughetto, E., Héry-Arnaud, G., Cariou, M. E., Pelloux, I., Maurin, M., Caillon, J., et al. (2015). An original case of Francisella tularensis subsp. holarctica bacteremia after a near-drowning accident. Infect. Dis. 47, 588-590. doi: 10. 3109/23744235.2015.1028099

Weinstein, M. P. (2003). Blood culture contamination: persisting problems and partial progress. J. Clin. Microbiol. 41, 2275-2278. doi: 10.1128/JCM.41.6.22752278.2003

Yu, J. J., Raulie, E. K., Murthy, A. K., Guentzel, M. N., Klose, K. E., and Arulanadam, B. P. (2008). The presence of infectious extracellular Francisella tularensis subsp. novicida in murine plasma after pulmonary challenge. Eur. J. Clin. Microbiol. Infect. Dis. 27, 323-325. doi: 10.1007/s10096-007-0434-x

Zahavy, E., Ber, R., Gur, D., Abramovich, H., Freeman, E., Maoz, S., et al. (2012). "Application of nanoparticles for the detection and sorting of pathogenic bacteria by flow-cytometry," in Nano-Biotechnology for Biomedical and Diagnostic Research, eds E. Zahavy, A. Ordentlich, S. Yitzhaki, and A. Shafferman (Dordrecht: Springer), 23-36.

Conflict of Interest Statement: The authors declare that the research was conducted in the absence of any commercial or financial relationships that could be construed as a potential conflict of interest.

Copyright (C) 2017 Aloni-Grinstein, Schuster, Yitzhaki, Aftalion, Maoz, SteinbergerLevy and Ber. This is an open-access article distributed under the terms of the Creative Commons Attribution License (CC BY). The use, distribution or reproduction in other forums is permitted, provided the original author(s) or licensor are credited and that the original publication in this journal is cited, in accordance with accepted academic practice. No use, distribution or reproduction is permitted which does not comply with these terms. 